\title{
Botulinum Toxin A Ameliorates Neuroinflammation in the MPTP and 6-OHDA-Induced Parkinson's Disease Models
}

\author{
Hyeon Joo Ham ${ }^{1, \dagger}$, In Jun Yeo, ${ }^{1, \dagger}$, Seong Hee Jeon ${ }^{1}$, Jun Hyung Lim, Sung Sik Yoo ${ }^{1}$, Dong Ju Son ${ }^{1}$, \\ Sung-Su Jang ${ }^{2}$, Haksup Lee ${ }^{2}$, Seung-Jin Shin ${ }^{2}$, Sang Bae Han ${ }^{1}$, Jae Suk Yun ${ }^{1}$ and Jin Tae Hong ${ }^{1, *}$ \\ ${ }^{1}$ College of Pharmacy and Medical Research Center, Chungbuk National University, Cheongju 28644, \\ ${ }^{2}$ ATGC Co., Seoul 06372, Republic of Korea
}

\begin{abstract}
Recently, increasing evidence suggests that neuroinflammation may be a critical factor in the development of Parkinson's disease $(P D)$ in addition to the ratio of acetylcholine/dopamine because dopaminergic neurons are particularly vulnerable to inflammatory attack. In this study, we investigated whether botulinum neurotoxin A (BoNT-A) was effective for the treatment of PD through its anti-neuroinflammatory effects and the modulation of acetylcholine and dopamine release. We found that BoNT-A ameliorated MPTP and 6-OHDA-induced PD progression, reduced acetylcholine release, levels of IL-1 $\beta$, IL-6 and TNF- $\alpha$ as well as GFAP expression, but enhanced dopamine release and tyrosine hydroxylase expression. These results indicated that BoNT-A had beneficial effects on MPTP or 6-OHDA-induced PD-like behavior impairments via its anti-neuroinflammation properties, recovering dopamine, and reducing acetylcholine release.
\end{abstract}

Key Words: Parkinson's disease, Botulinum toxin A, Anti-neuroinflammation

\section{INTRODUCTION}

Parkinson's disease (PD) is a common neurodegenerative disorder with an estimated worldwide prevalence of $0.4 \%$ to $2 \%$ in the elderly population (Pringsheim et al., 2014). PD is characterized by selective dopaminergic (DA) neuronal degeneration and loss of the dopamine neurotransmitter in nigrostriatal regions of the brain (Chaudhuri et al., 2006). Apart from typical motor symptoms (resting tremors, bradykinesia, and rigidity), non-motor symptoms such as cognitive impairment (attention deficits, memory difficulties, dementia), sleep difficulty, and psychiatric disorders (anxiety, depression, and psychosis) are also associated with PD. Furthermore, a decrease of DA is followed by an increase of acetylcholine (ACh) released from cholinergic striatal interneurons (Day et al., 2006). L-dopa therapy has been the mainstay of PD therapy since 1969 but it is limited by its efficacy and side effects (Marsden, 1982; Coffield and Yan, 2009). Moreover, many dopaminergic drugs cannot easily cross the blood-brain-barrier. Therefore, other treatment strategies such as aromatic amino acid decarboxylase, glutamic acid decarboxylase, and glial cell line-derived neurotrophic factor gene therapy have been suggested (Ding et al., 2006; Boll et al., 2011; Oldenburg and Ding, 2011). Treatment with anti-cholinergic drugs has been used to antagonize striatal hypercholinism in PD, but it often causes side effects including confusion, hallucinations, transient cognitive impairment, nausea, dry mouth, constipation, dizziness, and blurred vision (Boll et al., 2011; Connolly and Lang, 2014).

Several species of botulinum neurotoxin (BoNT) act on cholinergic terminals of the peripheral neuromuscular junction and the central nervous system (CNS) (Hawlitschka et al., 2013). BoNT causes the rapid inhibition of the voluntary nervous circuits by blocking the release of ACh (Rossetto et al., 2013). Type A BoNT have been classified into five subtypes (A1-A5) based on the amino acid sequence variability of BoNT. All subtypes bind to presynaptic synaptosomal-associated protein 25 (SNAP-25) with similar affinity, but BoNTA1 and BoNT-A2 have a greater cleavage effect on SNAP-25 than other subtypes. SNAP-25 cleaved by BoNT-A blocks the transmission of neuronal signals (Knuepfer and Juenemann, 2014). These characteristics have promoted the therapeutic testing of BoNT-A in several animal models of PD. Interruption of $\mathrm{ACh}$ release in the striatum by the direct injection of BoNT-A

\section{Open Access https://doi.org/10.4062/biomolther.2021.077}

This is an Open Access article distributed under the terms of the Creative Commons Attribution Non-Commercial License (http://creativecommons.org/licenses/by-nc/4.0/) which permits unrestricted non-commercial use, distribution, and reproduction in any medium, provided the original work is properly cited.
Received Apr 20, 2021 Revised May 13, 2021 Accepted May 17, 2021

Published Online Jun 3, 2021

\section{${ }^{*}$ Corresponding Author}

E-mail: jinthong@chungbuk.ac.kr

Tel: +82-43-261-2813, Fax: +82-43-268-2732

${ }^{\dagger}$ The first two authors contributed equally to this work. 
was reported in a rat unilateral 6-hydroxydopamine (6-OHDA) model of PD (Wree et al., 2011). In addition, the use of BoNTA to treat PD without adverse side effects such as memory dysfunction has been demonstrated (Antipova et al., 2013). Recent studies showed the clinical administration of BoNT-A to the brain was a therapeutic strategy for the treatment of neurological disorders such as blepharospasm, hemifacial spasm, and dystonia (Hawlitschka et al., 2013; Knuepfer and Juenemann, 2014).

As a progressive neurodegenerative disorder, PD is a multifactorial complex disease most likely caused by genetic and environmental risk factors, as well as cellular alterations and aging. The deregulation of inflammatory pathways resulting from genetic vulnerability as well as immune alterations associated with aging and the primary activation of glia by neuronal injury are also potentially involved in the pathogenesis of PD. Neuroinflammatory cytokines (interferon gamma (IFN- $\gamma$ ); tumor necrosis factor (TNF)- $\alpha$; interleukin (IL)-6; or IL-1 $\beta$ ) in affected brain regions of PD accumulated in the cerebrospinal fluid and post-mortem brain (Mogi et al., 1994). Numerous studies of experimental PD models indicated that dopamine neurons are particularly vulnerable to oxidative stress and inflammatory attack (Pott Godoy et al., 2008). Interestingly, lipopolysaccharide (LPS)-activated microglia in the vicinity of dopamine neurons in the substantia nigra (SN) induce the degeneration of these neurons whilst sparing GABAergic and serotonergic neurons, suggesting selective dopamine neuron vulnerability to inflammation (Liu and Bing, 2011). Although the application of BoNT-A to neuronal diseases has been demonstrated, the molecular mechanisms involved are not clear. Thus, in the present study, we investigated whether BoNT-A was effective for the treatment of PD through its antiinflammatory properties.

\section{MATERIALS AND METHODS}

\section{MPTP-induced PD model}

MPTP was used to establish the PD mice model. Each C57BL/6 mouse in MPTP group received intraperitoneal injection of MPTP $20 \mathrm{mg} / \mathrm{kg}$ four times in 7 days, while the same amount of normal saline was injected to each mouse in control group. In BoNT-A group, each mouse received 25 pg/mouse BoNT-A by ICV injection. MPTP+BoNT-A group mouse was treated with MPTP $(20 \mathrm{mg} / \mathrm{kg})$ four times in 7 days by intraperitoneal injection, followed by ICV injection of BoNT-A (25 $\mathrm{pg} /$ mouse) $24 \mathrm{~h}$ after MPTP treatment.

\section{6-OHDA-induced PD model}

C57BL/6 Mice were positioned on a stereotaxic frame (Stoelting Co., Wood Dale, IL, USA) and injected in the right stratum with $2 \mu \mathrm{L}$ of 6-OHDA hydrochloride (H4381; SigmaAldrich, St. Louis, MO, USA) (10 $\mu \mathrm{g} / \mathrm{mouse})$. The coordinates related to Bregma were AP: $+0.5 \mathrm{~mm}, \mathrm{ML}:-2.0 \mathrm{~mm}$ and $\mathrm{DV}$ : $-4.5 \mathrm{~mm}$ according to Paxinos and Watson (2006). Sham operated animals were submitted to the same procedure except $4 \mu \mathrm{L}$ vehicle ( $0.9 \%$ saline) was infused into the striatum instead of 6 OHDA. Two weeks after injection of 6-OHDA, BoNT-A (50 pg/mouse) was injected into the right straitum using stereotaxic apparatus. Before the surgical procedure, animals were intraperitoneally anesthetized with ketamine $(75 \mathrm{mg} / \mathrm{kg})$. During the surgery and recovery, animals were kept warm us- ing a heating pad. Three weeks after injection of BoNT-A, the behavior tests were performed. The protocols were reviewed and approved by the Chungbuk National University Institutional Animal Care and Use Committee (IACUC) and complied with the Korean National Institute of Health Guide for the Care and Use of Laboratory Animals (CBNUA-1117-18-01).

\section{Behavior tests}

Behavior changes were tested by Rota rod, Pole and gait tests as described elsewhere (Hwang et al., 2017). Motor performance and coordination were examined using the Rota rod treadmill (MED Associates Inc., St. Albans, VT, USA), consisting of a 3.6-cm diameter cylindrical treadmill connected to a computer controlled stepper motor as described previously (Choi et al., 2013). The pole test trials were performed using the rough-surfaced wooden pole three times per animal and average values from three examinations were used for each animal. A gait test trial was performed on a bright runway $(4.5 \mathrm{~cm}$ wide $)$ and a dark goal box $(20 \times 17 \times 10 \mathrm{~cm})$ and stride length was measured as the distance between successive paw prints. Data was presented as the average of five strides for each animal.

\section{Immunohistochemistry}

Immunocytochemical analysis was conducted as describe elsewhere (Hwang et al., 2017). Brain sections were transferred to $30 \%$ sucrose solutions, and then were cut into $20-\mu \mathrm{m}$ sections using a cryostat microtome (Leica CM 1850; Leica Microsystems, Seoul, Korea). After two washes in PBS (pH 7.4) for $10 \mathrm{~min}$ each, the samples were incubated with $3 \%$ hydrogen peroxide and $1 \%$ Triton-X in PBS for 30 min for antigen retrieval and blocking of endogenous peroxidase. After an additional two washes in PBS for 10 min each, the brain sections were blocked for $1 \mathrm{~h}$ in $3 \%$ bovine serum albumin (BSA) solution and incubated overnight at $4{ }^{\circ} \mathrm{C}$ with glial fibrillary acidic protein (GFAP; 1:300; Santa Cruz Biotechnology, Inc., Santa Cruz, CA, USA), and anti-tyrosine hydroxylase (TH; 1:300, Cell Signaling Mol Neurobiol Technology, Inc., Beverly, MA, USA). Brain sections were then washed three times in PBS for 10 min each, and were incubated for 1-2 $\mathrm{h}$ at room temperature with the biotinylated goat anti-rabbit, goat anti-mouse, or donkey anti-goat IgG-horseradish peroxidase (HRP) secondary antibodies (1:500; Santa Cruz Biotechnology, Inc.). After washing three times in PBS for 10 min each, and then visualized by a chromogen diaminobenzidine (Vector Laboratories, Burlingame, CA, USA) reaction for up to $10 \mathrm{~min}$. Finally, brain sections were dehydrated in ethanol, cleared in xylene, mounted with Permount (Fisher Scientific, Hampton, $\mathrm{NH}, \mathrm{USA}$ ), and evaluated on a light microscope (Microscope Axio Imager. A2; Carl Zeiss, Oberkochen, Germany; $\times 50$ and $\times 200$ ).

\section{Western blot analysis}

The brain tissues were homogenized with lysis buffer (PROPREP; iNtRON, Sungnam, Korea; $n=8$ mice per group) and centrifuged at $2,500 \times \mathrm{g}$ for $15 \mathrm{~min}$ at $4^{\circ} \mathrm{C}$. Equal amounts of total protein $(40 \mu \mathrm{g})$ isolated from brain tissues were resolved on 8 or $10 \%$ sodium dodecyl sulfate polyacrylamide gels and then transferred to nitrocellulose membranes $(\mathrm{Hy}$ bond ECL; Amersham Pharmacia Biotech, Piscataway, NJ, USA). Membranes were incubated at roomtemperature for 2 $\mathrm{h}$ with anti-Tyrosine hydroxylase (Cell Signaling Mol Neurobiol 
Technology, Inc.), anti-GFAP (1:1,000; Santa Cruz Biotechnology, Inc.) and anti- $\beta$-actin (1:2,500; Sigma-Aldrich). Blots were then incubated at room temperature for $2 \mathrm{~h}$ with, corresponding peroxidase-conjugated anti-mouse or anti-rabbit antibodies (1:2,000; Santa Cruz Biotechnology, Inc.). Immunoreactive proteins were detected using an enhanced chemiluminescence Western blotting detection system. The relative density of the protein bands was scanned densitometrically using My Image (SLB, Seoul, Korea) and quantified by Lab Works 4.0 (UVP, Upland, CA, USA).

\section{Measurement of dopamine and acetylcholine}

Lysates of whole brain tissue were obtained through protein extraction buffer containing protease inhibitor. Dopamine and acetylcholine levels were determined using each specific ELISA Kit (Wuhan Huamei Biotech Co., Ltd., Hubei, China). In brief, $100 \mu \mathrm{L}$ of sample was added into the precoated plate and incubated for overnight at $4^{\circ} \mathrm{C}$. After washing each well of the precoated plate with washing buffer, $100 \mu \mathrm{L}$ of labeled antibody solution was added, and the mixture was incubated for $1 \mathrm{~h}$ at $4^{\circ} \mathrm{C}$ in the dark. After washing, chromogen was added, and the mixture was incubated for $30 \mathrm{~min}$ at room temperature in the dark. Finally, the resulting color was assayed at 450 $\mathrm{nm}$ (570 nm for acetylcholine) using a microplate absorbance reader (SunriseTM, TECAN, Männedorf, Switzerland) after adding stop solution.

\section{Levels of TNF- $\alpha$, IL-1 $\beta$, and IL- 6 in the brain}

The levels of TNF- $\alpha, \mathrm{IL}-1 \beta$, and IL- 6 in brain were measured by RT-PCR. Total RNA was extracted using RiboEX (Geneall biotechnology, Seoul, Korea) from hippocampus tissue and cDNA was synthesized using High-Capacity cDNA Reverse Transcription kit (Thermo Scientific, Waltham, MA, USA). Quantitative real-time PCR was performed on a 7,500 real-time PCR system (Applied Biosystems, Foster City, CA, USA) for custom-designed primers and $\beta$-actin was used for house-keeping control using HiPi Real-Time PCR SYBR green master mix (ELPIS biotech, Daejeon, Korea). Cycling conditions consisted of an initial denaturation step of 3 min at $94^{\circ} \mathrm{C}$, a denaturation step of $30 \mathrm{~s}$ at $94^{\circ} \mathrm{C}$, an annealing step of $30 \mathrm{~s}$ at $60^{\circ} \mathrm{C}$ and an extension step of a minute at $72^{\circ} \mathrm{C}$ fol- lowed by 40 cycles. The values obtained for the target gene expression were normalized to $\beta$-actin and quantified relative to the expression in control samples.

Each sample was run with the following primer pairs: $\beta$-actin, Forward primer: 5'- GGCTGTATTCCCCTCCATCG-3', Reverse primer: 5'- CCAGTTGGTAACAATGCCATGT-3'; TNF- $\alpha$, Forward primer: 5'-TCTTCTCATTCCTGCTTGTGG-3', Reverse primer: 5'- CACTTGGTGGTTTGCTACGA-3'; IL-1 $\beta$, Forward primer: 5'-CCTTCCAGGATGAGGACATGA-3', Reverse primer: 5'-TGAGTCACAGAGGATGGGCTC-3'; IL-6, Forward primer: 5'-GAGGATACCACTCCCAACAGACC-3', Reverse primer: 5'-AAGTGCATCATCGTTGTTCATACA-3'.

\section{Statistical analysis}

GraphPad Prism 4 software (GraphPad Software, La Jolla, CA, USA) was used for statistical analysis. A one-way analysis of variance (ANOVA) was applied for assessment of difference among the graphs. Once the significant was found, the differences were further analyzed by the Dunnett's test. Data are presented as mean $\pm S D$, a value of $p<0.05$ was considered to be statistically significant.

\section{RESULTS}

\section{Effects of BoNT-A on behavioral impairments induced by MPTP and 6-OHDA}

We evaluated the survival rate of PD mice after BoNT-A application. Survival rate was measured at 1 and 3 days after 50,25 , or 12.5 pg BoNT-A injection by intraceverbrventricu$\operatorname{lar}(\mathrm{ICV})$ injection. Treatment with $50 \mathrm{pg}$ BoNT-A resulted in a significant loss of survival at $48 \mathrm{~h}$ compared with the 25 and $12.5 \mathrm{pg}$ treatment groups (data not shown). Therefore, we administered $25 \mathrm{pg} / \mathrm{mouse}$ of BoNT-A in all future experiments. We examined the effect of BoNT-A on behavioral disorders induced by MPTP. The rota-rod test was conducted to test coordination capability. The MPTP treated group had significantly decreased latency on the rota-rod. However, $24 \mathrm{~h}$ after treatment there was no difference between the MPTP and MPTP+BoNT-A treated groups (data not shown). However, after $72 \mathrm{~h}$, the latency in the MPTP+BoNT-A (25 pg/mouse)
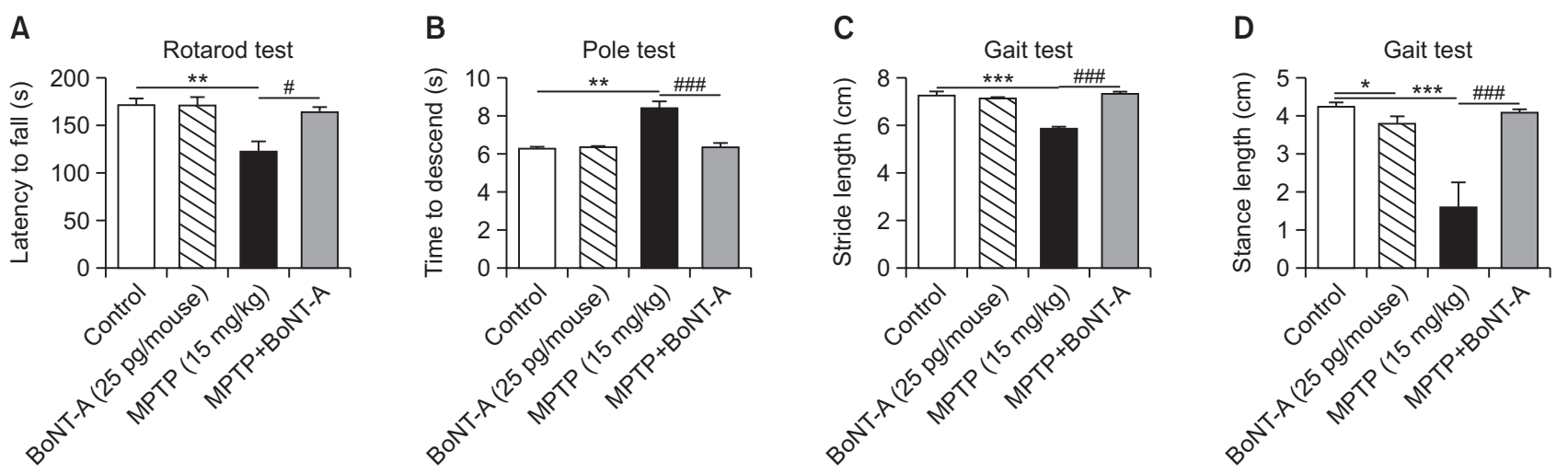

Fig. 1. Effect of BoNT-A on MPTP-induced behavior disorder. MPTP induced behavioral impairments. Performance on the rotarod was impaired by MPTP in mice (A). MPTP also induced bradykinesia in mice (B). Stride length of forelimb (C) and Stance length (D) were also shortened by MPTP injection which were recovered with BoNT-A treatment. Each value is presented as mean \pm SD from 8 mice. ${ }^{*} p<0.05$, ${ }^{* *} p<0.01$, and ${ }^{* * *} p<0.001$ significant difference from saline-injected mice and ${ }^{\#} p<0.05$ and ${ }^{\# \#} p<0.001$ significant difference from MPTP treated mice. 
co-treated mice significantly increased compared with that in the MPTP treated mice (Fig. 1A). Next, we performed the pole test, which measures the time to descend from the top of a pole to the floor, and is considered to reflect bradykinesia. The vehicle and BoNT-A treated groups did not show any significant differences related to behavioral disorders. However, the time to descend was significantly decreased in the MPTP+BoNT-A treated mice compared with the MPTP treated mice (Fig. 1B). In the gait test, the stride and stance lengths of the hind limb were significantly increased in the MPTP+BoNT-A co-treated mice than in the MPTP treated mice (Fig. 1C, 1D). However, there was no significant difference in terms of behavioral dis- orders between MPTP treated mice and MPTP+BoNT-A cotreated mice. We confirmed this recovery effect of BoNT-A in 6-OHDA treated mice. In the 6-OHDA model, we used $50 \mathrm{pg} /$ mouse of BoNT-A because the injection of BoNT-A through a stereotaxic apparatus did not cause death. The recovery effects were reproduced by BoNT-A in the 6-OHDA model even though 6-OHDA caused severely impaired coordination capability (Fig. 2A) and bradykinesia (Fig. 2B); however, there was a significant improved in gait test (Fig. 2C, 2D).

\section{Effects of BoNT-A on DA and ACh levels}

MPTP reduces dopamine levels but increases ACh (ratio
A

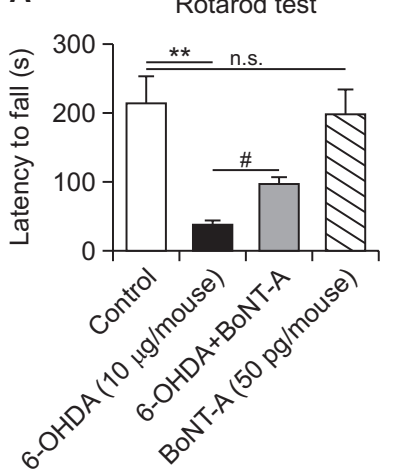

B

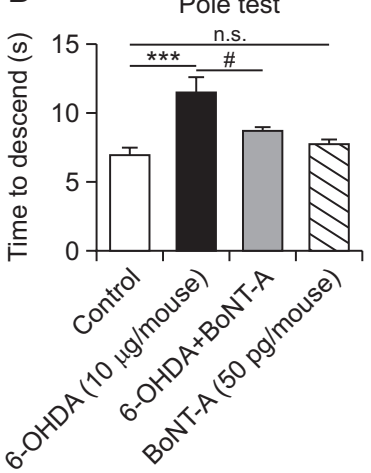

C

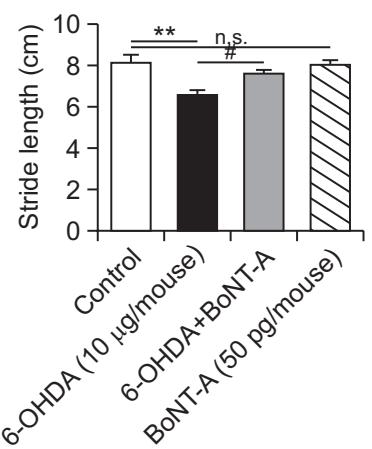

D

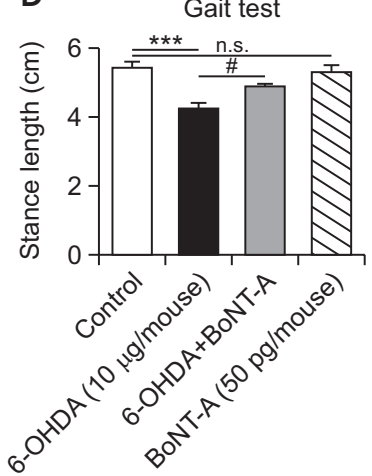

Fig. 2. Effect of BoNT-A on 6-OHDA-induced behavior disorder. 6-OHDA induced behavioral impairments. Performance on the rotarod was impaired by MPTP in mice (A). 6-OHDA also induced bradykinesia in mice (B). Stride length of forelimb (C) and Stance length (D) were also shortened by 6 -OHDA injection which were recovered with BoNT-A treatment. Each value is presented as mean \pm SD from 8 mice. ${ }^{* *} p<0.01$ and ${ }^{* * *} p<0.001$ significant difference from saline-injected mice and ${ }^{*} p<0.05$ significant difference from 6-OHDA treated mice.
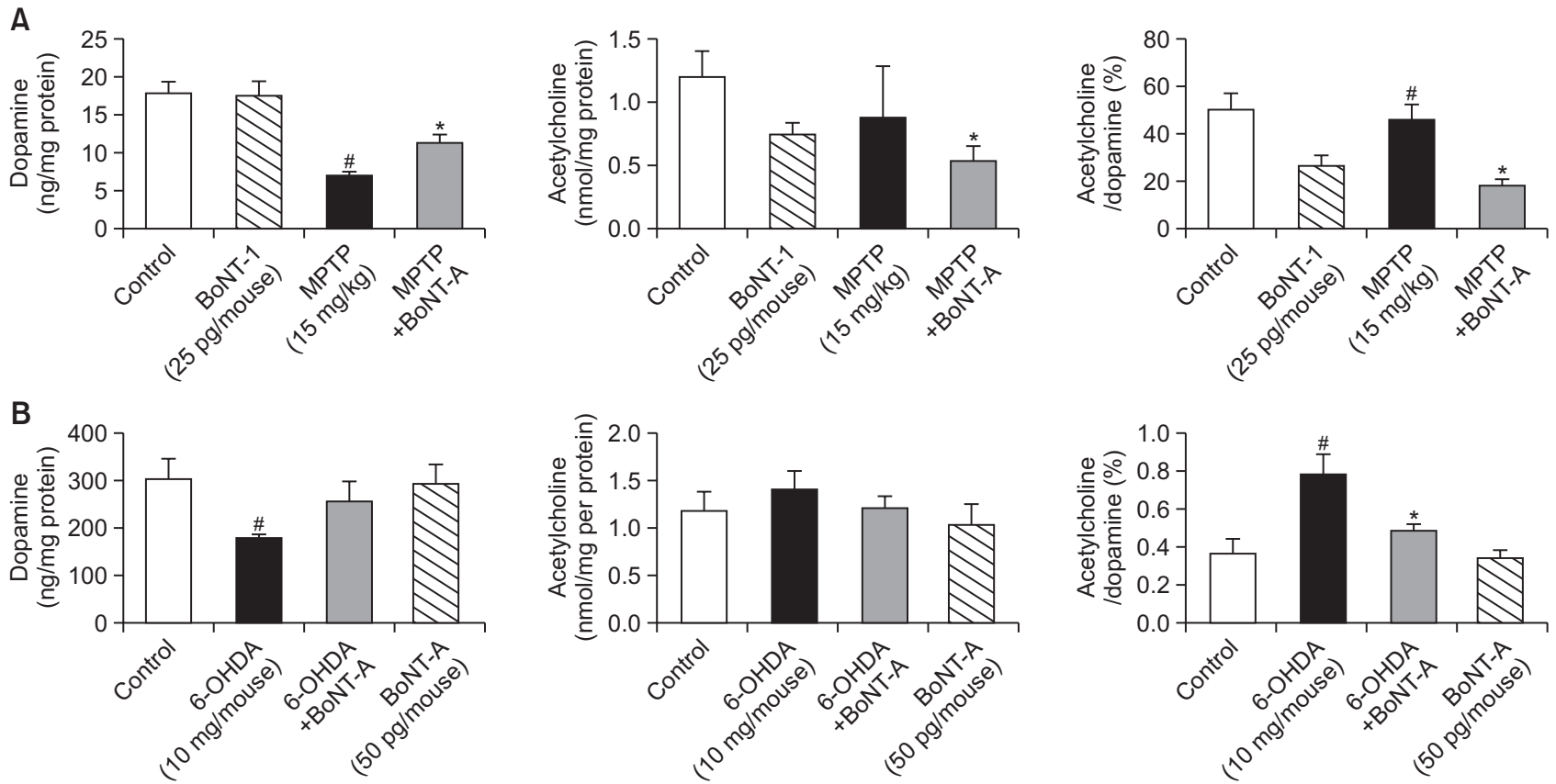

Fig. 3. Effect of BoNT-A on dopamine and acetylcholine levels by MPTP or 6-OHDA. Level of dopamine (left panel), acetylcholine (middle panel) and its ratio (right panel) in the MPTP or 6-OHDA-injected mice brain were determined by ELISA assay. Method was as described detail in materials and methods section. The results are expressed as mean \pm SD of 8 mice. ${ }^{*} p<0.05$ significant difference from saline-injected mice and ${ }^{\#} p<0.05$ significant difference between the MPTP or 6-OHDA treated mice. 
is more important), which are representative characteristics of PD. Because BoNT A inhibits ACh release, we examined whether BoNT A-mediated changes in the DA and ACh levels in MPTP- or 6-OHDA-treated mice are related to their behavioral changes. The level of DA was reduced by MPTP and recovered by BoNT-A treatment. There was no change in the level of ACh in the MPTP treated group brain tissues, although it was significantly reduced by BoNT-A treatment. Moreover, the significantly elevated ACh/DA ratio was reduced by BoNT-A (Fig. 3A). Furthermore, 6-OHDA treatment significantly reduced DA levels and BoNT-A significantly recovered the DA levels (Fig. 3B). However, the ACh level did not change with 6-OHDA treatment. The ACh/DA ratio was elevated by $6-\mathrm{OHDA}$ but reduced by the combination treatment of 6-OHDA+BoNT-A (Fig. 3B).

Dopaminergic neuronal cell death is implicated in the development of PD. Thus, we determined the TH positive cell number and $\mathrm{TH}$ expression. Abundant $\mathrm{TH}$-positive fibers in the striatum were found in the control and BoNT-A treated groups, whereas the number was significantly lower in the striatum of the MPTP treated group mouse brains, although BoNT-A treatment recovered this (Fig. 4A, upper IHC panel). The expression of TH was decreased by MPTP (Fig. 4B, upper WB panel) and its expression was recovered by BoNT-A treatment. Similar to MPTP treatment, abundant TH-positive fibers in the substantia nigra were found in the control and BoNT-A treated groups, whereas the number of TH-positive neurons was significantly lowered in the substantia nigra of the 6-OHDA treated group mouse brains. However, BoNT-A treatment significantly recovered the reduced number of $\mathrm{TH}$ positive neurons in 6-OHDA treated mice (Fig. 5A, upper IHC panel). The expression patterns of $\mathrm{TH}$ were similar to the change in TH cell number in the MPTP and 6-OHDA treated groups with/without BoNT-A treatment (Fig. 4B, 5B, upper panel). These results indicated that the recovery effects of BoNT-A on MPTP or 6-OHDA induced behavioral impairments were related to the recovery of DA and the reducing effect on ACh release.
A

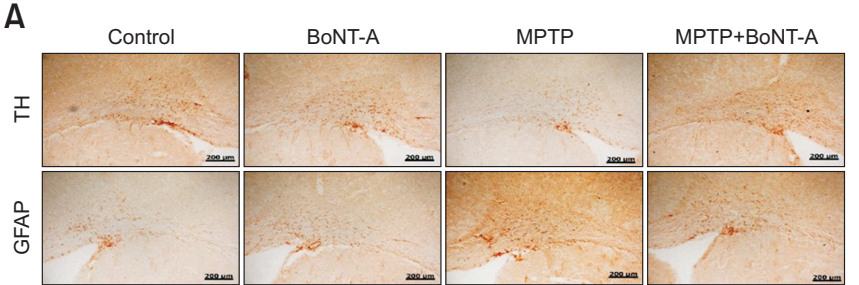

B

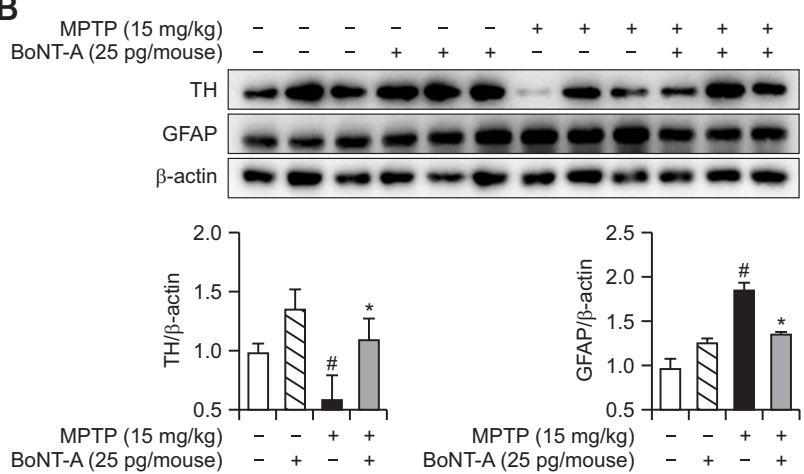

Fig. 4. Effect of BoNT-A on MPTP -induced on the expression of TH and GFAP. Tyrosine hydroxylase (TH) and activation of astrocytes (GFAP) was measured by immunohistochemical analysis and Western blotting analysis. The sections of mice brain (substantia nigra) incubated with anti-GFAP primary antibody and the biotinylated secondary antibody $(n=3)$. The represented stained tissues were viewed with a microscope $(\times 50$ or $\times 200)(A)$. Expression of GFAP was also examined by Western blotting with specific antibodies in the substantia nigra of brain (B). The graph represents Arbitrary density of each blots. All values are the means \pm SD from three mice brains. ${ }^{*} p<0.05$ significant difference from saline-injected mice and ${ }^{\#} p<0.05$ significant difference between the MPTP treated mice.

A

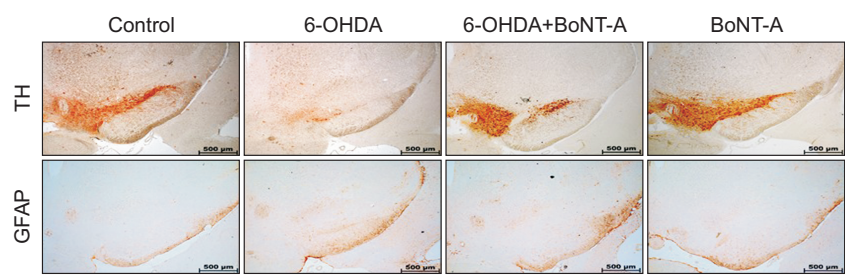

B $\begin{array}{cccccccccccccc}\text { 6-OHDA }(10 \mathrm{mg} / \mathrm{mouse}) & - & - & - & - & - & - & + & + & + & + & + & + \\ \text { BoNT-A }(50 \mathrm{pg} / \mathrm{mouse}) & - & - & - & + & + & + & - & - & - & + & + & +\end{array}$ BoNT-A (50 pg/mouse) -
TH GFAP $\beta$-actin

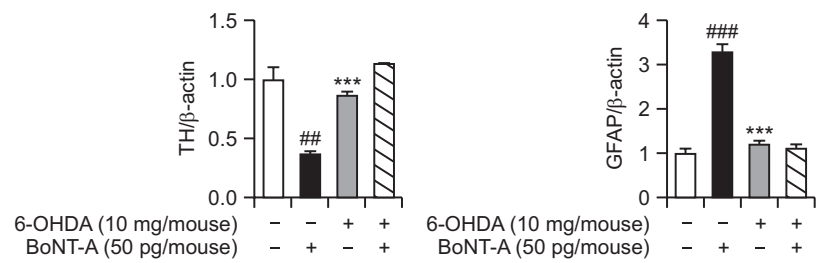

Fig. 5. Effect of BoNT-A on 6-OHDA-induced on the expression of TH and GFAP. The effect of estrogen on reactive astrocytes was measured by immunohistochemical analysis and Western blotting analysis. The sections of mice brain (striatum) incubated with anti-GFAP primary antibody and the biotinylated secondary antibody $(n=3)$. The represented stained tissues were viewed with a microscope $(\times 50$ or $\times 200)(A)$. Expression of GFAP was also examined by Western Blotting with specific antibodies in the brain (B). The graph represents Arbitrary density of each blots. All values are the means \pm SD from three mice brains. ${ }^{* * *} p<0.001$ significant difference from saline-injected mice and ${ }^{\# \#} p<0.01$, and ${ }^{\# \#} p<0.001$ significant difference from 6-OHDA treated mice. 
A
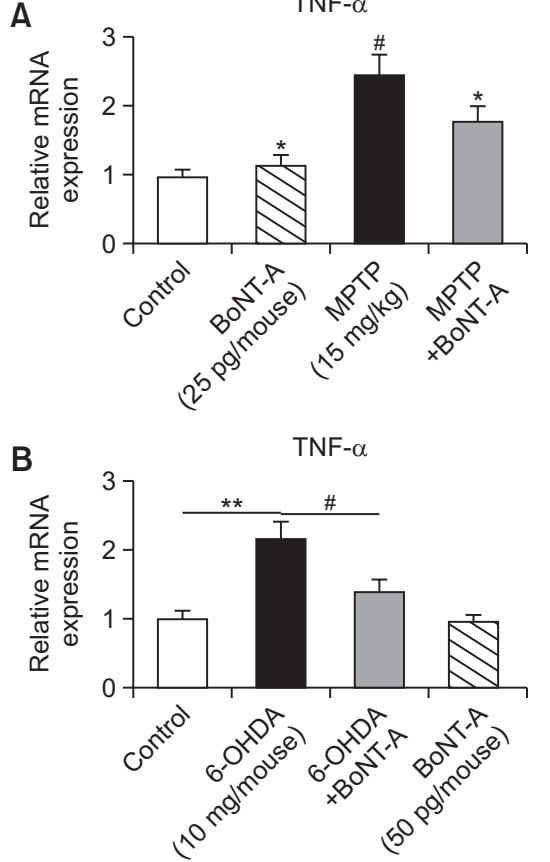

$\mathrm{IL}-1 \beta$
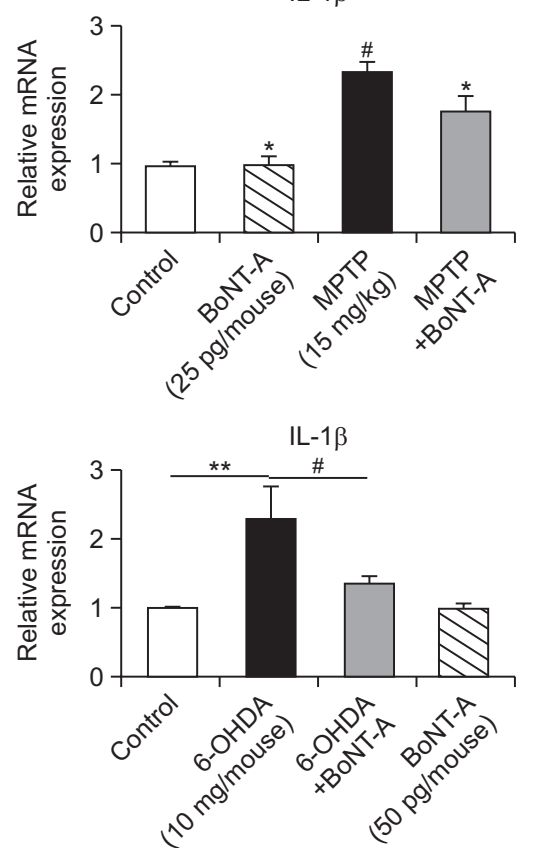

IL-6
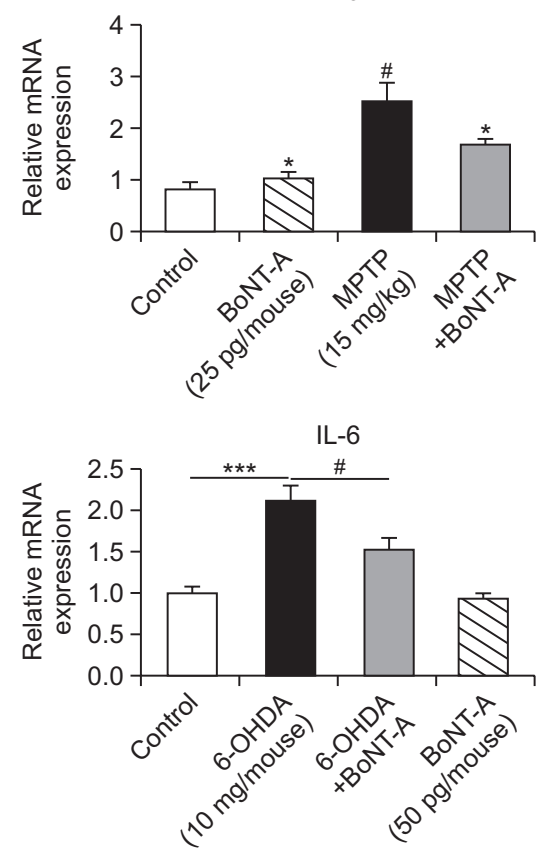

Fig. 6. Effect of BoNT-A on cytokine levels by MPTP or 6-OHDA. Level of TNF- $\alpha$ (left panel), IL-1 $\beta$ (middle panel) and IL-6 (right panel) in the MPTP (A) or 6-OHDA (B)-injected mice whole brains were determined by RT-PCR. Method was as described detail in materials and methods section. The results are expressed as mean \pm SD of 8 mice. ${ }^{*} p<0.05$, ${ }^{* *} p<0.01$, and ${ }^{* * *} p<0.001$ significant difference from salineinjected mice and ${ }^{\#} p<0.05$ significant difference between the MPTP or 6-OHDA treated mice.

\section{Effect of BoNT-A on neuroinflammation}

Astrocytes play important roles in neuroinflammation, which is a key factor in dopaminergic neurodegeneration. To determine whether MPTP or 6-OHDA injection induces neuroinflammation and the activation of astrocytes, we compared the expression of GFAP by WB and IHC as well as cytokine levels in the brains of MPTP or 6-OHDA mice with/without BoNT-A. The results revealed that the GFAP protein levels and positive cell numbers were significantly increased in MPTP (Fig. 4A, 4B, lower panel) and 6-OHDA treated groups (Fig. 5A, 5B, lower panel). However, the GFAP positive cell numbers and protein levels were significantly inhibited by BoNT-A (Fig. 4, 5). Next, we measured the cytokine levels in whole brain by RTPCR. MPTP (Fig. 6A) and 6-OHDA (Fig. 6B) elevated TNF- $\alpha$, IL-1 $\beta$, and IL- 6 levels, which were significantly reduced by BoNT-A treatment.

\section{DISCUSSION}

The application of BoNT-A to PD has been demonstrated in several studies, but the molecular mechanisms are not clear. In the present study, we found that BoNT-A was therapeutically effective in two mouse PD models, and the DA level and ACh/DA ratio were recovered to control levels. These effects were associated with reduced cytokine levels and the inactivation of astrocytes and microglia.

It is noteworthy that neuroinflammatory cytokines including IFN- $\gamma$, TNF- $\alpha$, IL- 6 , and IL- $1 \beta$ accumulate in the cerebrospinal fluid and postmortem brains of PD patients (Mogi et al., 1994). Furthermore, TNF- $\alpha, \mathrm{IL}-6, \mathrm{IL}-1 \beta$, and IL-10 were reported to control the biological neuroinflammation processes underlying
PD as analyzed using the STRING and CentiScaPe proteinnetwork analysis program (Bonte et al., 2021). In this regard, the role of neuroinflammation is important in the progression of $P D$, and it is interesting that several anti-diabetes drugs including GLP-1 and a GIP receptor agonist are neuroprotective in a mouse model of Parkinson's disease by preventing neuroinflammation (Zhang et al., 2021a, 2021b). These in vivo animal data, patient data with big data support, and our data, show that BoT significantly ameliorated the progression of PD through the inhibition of neuroinflammation in PD animal models.

BoT has been widely used to treat muscle spasm, dystonia, spasticity, and myofascial pain syndrome (Jankovic, 2004). In addition, BoT was also reported to improve radiation fibrosis syndrome, inhibit the production of collagen during urethral wound healing, retard fibrosis, and treat frozen shoulder (Stubblefield et al., 2008; Sahinkanat et al., 2009). Furthermore, the intra-articular injection of BoT reduced neurogenic inflammation in a complete Freund's adjuvant (CFA)induced arthritis rat model (Wang et al., 2017). Even though the molecular mechanism of the anti-inflammatory effects of BoT remains unclear, the intra-temporomandibular Joint Injection (TMJ) of BoNT-A may promote a central effect by reducing the P2X7 receptor/Cathepsin S (CatS)/Fractalkine (FKN) microglia-activated pathway in the trigeminal subnucleus caudalis (Muñoz-Lora et al., 2020). In addition, BoT modulated spinal neuron-glia interactions under neuropathic pain conditions (Rojewska et al., 2018). BoT also reduced LPS-induced neuroinflammation by dampening the activation of microglial cells and astroglial activity (Piotrowska et al., 2017). These and our results suggest that the protective roles of BoT on these diseases may be associated with anti-inflammatory ef- 
fects related to the inhibition of astrocyte and microglia activities.

Astrocytic and oligodendrocytic $\mathrm{P} 2 \mathrm{X} 7$ receptors mediate some of important neuronal functions in the CNS. For example, they mediate necrosis/apoptosis by releasing proinflammatory cytokines/chemokines, reactive oxygen species (ROS), and the excitotoxic (glio) transmitters glutamate and ATP (Liu and Bing, 2011; Zhao et al., 2021). The microglial phagocytosis of neurons causes neurodegenerative diseases including PD (Butler et al., 2021). Numerous studies of experimental PD models indicated that DA neurons are particularly vulnerable to immune and (or) inflammatory attacks (Pott Godoy et al., 2008). Interestingly, in this regard, LPS-activated microglia in the vicinity of DA neurons in the $S N$ induces the degeneration of DA neurons whilst sparing GABAergic and serotonergic neurons, suggesting a selective dopamine neuron vulnerability to inflammation. In our study, we found that MPTP and 6-OHDA induced lowers level of DA and ACh/DA ratio was recovered to control levels by BoT treatment. These effects were associated with reduced cytokine levels and the inactivation of astrocytes and microglia. Conclusively, our data indicated that BoT might ameliorate PD progression by recovering DA levels by inhibiting neuroinflammation.

\section{CONFLICT OF INTEREST}

The authors declare that they have no conflict of interest.

\section{ACKNOWLEDGMENTS}

This work is financially supported by the National Research Foundation of Korea [NRF] Grant funded by the Korea government (MSIP) (No. MRC, 2017R1A5A2015541).

\section{REFERENCES}

Antipova, V., Hawlitschka, A., Mix, E., Schmitt, O., Drager, D., Benecke, R. and Wree, A. (2013) Behavioral and structural effects of unilateral intrastriatal injections of botulinum neurotoxin a in the rat model of Parkinson's disease. J. Neurosci. Res. 91, 838-847.

Boll, M. C., Alcaraz-Zubeldia, M. and Rios, C. (2011) Medical management of Parkinson's disease: focus on neuroprotection. Curr. Neuropharmacol. 9, 350-359.

Bonte, M.-A., El Idrissi, F., Gressier, B., Devos, D. and Belarbi, K. (2021) Protein network exploration prioritizes targets for modulating neuroinflammation in Parkinson's disease. Int. Immunopharmacol. 95, 107526.

Butler, C. A., Popescu, A., Kitchener, E., Allendorf, D. H., Puigdellívol, M. and Brown, G. C. (2021) Microglial phagocytosis of neurons in neurodegeneration, and its regulation. J. Neurochem. doi: 10.1111/ jnc.15327 [Online ahead of print].

Chaudhuri, K. R., Healy, D. G. and Schapira, A. H. (2006) Non-motor symptoms of Parkinson's disease: diagnosis and management. Lancet Neurol. 5, 235-245.

Choi, D. Y., Lee, M. K. and Hong, J. T. (2013) Lack of CCR5 modifies glial phenotypes and population of the nigral dopaminergic neurons, but not MPTP-induced dopaminergic neurodegeneration. Neurobiol. Dis. 49, 159-168.

Coffield, J. A. and Yan, X. (2009) Neuritogenic actions of botulinum neurotoxin A on cultured motor neurons. J. Pharmacol. Exp. Ther. 330, 352-358.

Connolly, B. S. and Lang, A. E. (2014) Pharmacological treatment of
Parkinson disease: a review. JAMA 311, 1670-1683.

Day, M., Wang, Z., Ding, J., An, X., Ingham, C. A., Shering, A. F., Wokosin, D., Ilijic, E., Sun, Z. and Sampson, A. R. (2006) Selective elimination of glutamatergic synapses on striatopallidal neurons in Parkinson disease models. Nat. Neurosci. 9, 251-259.

Ding, J., Guzman, J. N., Tkatch, T., Chen, S., Goldberg, J. A., Ebert, P. J., Levitt, P., Wilson, C. J., Hamm, H. E. and Surmeier, D. J. (2006) RGS4-dependent attenuation of M 4 autoreceptor function in striatal cholinergic interneurons following dopamine depletion. Nat. Neurosci. 9, 832-842.

Hawlitschka, A., Antipova, V., Schmitt, O., Witt, M., Benecke, R., Mix, E. and Wree, A. (2013) Intracerebrally applied botulinum neurotoxin in experimental neuroscience. Curr. Pharm. Biotechnol. 14, 124-130

Hwang, C. J., Kim, Y. E., Son, D. J., Park, M. H., Choi, D. Y., Park, P. H., Hellstrom, M., Han, S. B., Oh, K. W., Park, E. K. and Hong, J. T. (2017) Parkin deficiency exacerbate ethanol-induced dopaminergic neurodegeneration by P38 pathway dependent inhibition of autophagy and mitochondrial function. Redox. Biol. 11, 456-468.

Jankovic, J. (2004) Botulinum toxin in clinical practice. J. Neurol. Neurosurg. Psychiatry 75, 951-957.

Knuepfer, S. and Juenemann, K. P. (2014) Experience with botulinum toxin type $A$ in the treatment of neurogenic detrusor overactivity in clinical practice. Ther. Adv. Urol. 6, 34-42.

Liu, M. and Bing, G. (2011) Lipopolysaccharide animal models for Parkinson's disease. Parkinsons Dis. 2011, 327089.

Marsden, C. (1982) The mysterious motor function of the basal ganglia: the Robert Wartenberg Lecture. Neurology 32, 514-539.

Mogi, M., Harada, M., Kondo, T., Riederer, P., Inagaki, H., Minami, M. and Nagatsu, T. (1994) Interleukin-1 beta, interleukin-6, epidermal growth factor and transforming growth factor-alpha are elevated in the brain from parkinsonian patients. Neurosci. Lett. 180, 147-150.

Muñoz-Lora, V. R. M., Abdalla, H. B., Cury, A. A. D. B. and ClementeNapimoga, J. T. (2020) Modulatory effect of botulinum toxin type A on the microglial P2X7/CatS/FKN activated-pathway in antigeninduced arthritis of the temporomandibular joint of rats. Toxicon 187, 116-121.

Oldenburg, I. A. and Ding, J. B. (2011) Cholinergic modulation of synaptic integration and dendritic excitability in the striatum. Curr. Opin. Neurobiol. 21, 425-432.

Paxinos, G. and Watson, C. (2006) The Rat Brain in Stereotaxic Coordinates. Hard cover ed. Elsevier.

Piotrowska, A., Popiolek-Barczyk, K., Pavone, F. and Mika, J. (2017) Comparison of the expression changes after botulinum toxin type $A$ and minocycline administration in lipopolysaccharide-stimulated rat microglial and astroglial cultures. Front. Cell. Infect. Microbiol. 7, 141.

Pott Godoy, M. C., Tarelli, R., Ferrari, C. C., Sarchi, M. I. and Pitossi, F. J. (2008) Central and systemic IL-1 exacerbates neurodegeneration and motor symptoms in a model of Parkinson's disease. Brain 131, 1880-1894.

Pringsheim, T., Jette, N., Frolkis, A. and Steeves, T. D. (2014) The prevalence of Parkinson's disease: a systematic review and metaanalysis. Mov. Disord. 29, 1583-1590.

Rojewska, E., Piotrowska, A., Popiolek-Barczyk, K. and Mika, J. (2018) Botulinum toxin type A-a modulator of spinal neuron-glia interactions under neuropathic pain conditions. Toxins 10, 145.

Rossetto, O., Megighian, A., Scorzeto, M. and Montecucco, C. (2013) Botulinum neurotoxins. Toxicon 67, 31-36.

Sahinkanat, T., Ozkan, K. U., Cıralık, H., Ozturk, S. and Resim, S. (2009) Botulinum toxin-A to improve urethral wound healing: an experimental study in a rat model. Urology 73, 405-409.

Stubblefield, M. D., Levine, A., Custodio, C. M. and Fitzpatrick, T. (2008) The role of botulinum toxin type $A$ in the radiation fibrosis syndrome: a preliminary report. Arch. Phys. Med. Rehabil. 89, 417421.

Wang, L., Wang, K., Chu, X., Li, T., Shen, N., Fan, C., Niu, Z., Zhang, $X$. and Hu, L. (2017) Intra-articular injection of Botulinum toxin A reduces neurogenic inflammation in CFA-induced arthritic rat model. Toxicon 126, 70-78.

Wree, A., Mix, E., Hawlitschka, A., Antipova, V., Witt, M., Schmitt, O. and Benecke, R. (2011) Intrastriatal botulinum toxin abolishes 
Ham et al. Botulinum Toxin A Improves Parkinson's Disease

pathologic rotational behaviour and induces axonal varicosities in the 6-OHDA rat model of Parkinson's disease. Neurobiol. Dis. 41 291-298.

Zhang, L. Y., Jin, Q. Q., Hölscher, C. and Li, L. (2021a) Glucagonlike peptide-1/glucose-dependent insulinotropic polypeptide dual receptor agonist DA-CH5 is superior to exendin-4 in protecting neurons in the 6-hydroxydopamine rat Parkinson model. Neural Regen. Res. 16, 1660-1670.
Zhang, Z., Hao, L., Shi, M., Yu, Z., Shao, S., Yuan, Y., Zhang, Z. and Hölscher, C. (2021b) Neuroprotective effects of a GLP-2 analogue in the MPTP Parkinson's disease mouse model. J. Parkinsons Dis. 11, 529-543.

Zhao, Y. F., Tang, Y. and Illes, P. (2021) Astrocytic and oligodendrocytic $\mathrm{P} 2 \mathrm{X} 7$ receptors determine neuronal functions in the CNS. Front. Mol. Neurosci. 14, 641570. 